\title{
Estructura de mercado del sistema bancario ecuatoriano: concentración y poder de mercado
}

\author{
Market structure in the Ecuadorian financial \\ system: concentration and power market
}

Segundo Camino-Mogro

Superintendencia de Compañías

Segundo.camino@gmail.com
Carolina Uzcátegui-Sánchez Universidad Metropolitana

(Ciudad - Pais)

cuzcategui@umet.edu.ec

Julio Moran-Cruz Investigador Independiente Giulio_cesar90@yahoo.es
Revista Cumbres Vol.4 №1

Versión impresa ISSN 1390-9541

Versión electrónica ISSN 1390-3365

http://investigacion.utmachala.edu.ec/revistas/index.php/Cumbres 


\title{
RESUMEN
}

Determinar el ambiente en el que compiten los bancos privados en Ecuador ayudaría a implementar políticas públicas de regulación bancaria, por lo que el presente trabajo analiza la estructura y competencia en el sector bancario en Ecuador desde el año 2000 hasta el 2015, enfocándose específicamente en dos segmentos: cartera y depósitos. Por tanto los objetivos que plantea este estudio son dos: 1) analizar la evolución de la estructura de mercado y 2) determinar el nivel de competencia y de concentración del mercado. Para analizar la estructura del mercado y poder determinar el grado de competencia se utilizan el indicador de concentración 4 y el índice Herfindahl-Hirschman de 34 bancos privados por medio de la información pública de la Superintendencia de Bancos del Ecuador. Como principales conclusiones se puede mencionar las siguientes: i) existe evidencia de que el mercado bancario en Ecuador posee una estructura oligopolística, ii) el sector bancario operaria como si existiesen 7 empresas en todo el mercado, y se las podría clasificar en bancos grandes y medianos y iii) los resultados del índice de Herfindahl-Hirschman muestran una concentración moderada a lo largo del periodo analizado en cartera y en depósitos, lo que no descarta la opción que los 4 bancos más grandes puedan ejercer cierto poder de mercado.

Palabras clave: Cncentración de mercado, competencia, bancos, poder de mercado, depósitos, créditos.

\begin{abstract}
Determining the environment in which private banks compete in Ecuador would help to implement public policies for banking regulation. In this sense, this work analyzes the structure and competition in the banking sector in Ecuador from 2000 to 2015, focusing specifically on loans and deposits. Therefore, the objectives raised for this study are 1) to analyze the evolution of the market structure and 2) to measure the level of competition and concentration in the market. In order to analyze the market structure and to determine the degree of competition, the concentration 4 index and Herfindahl-Hirschman index of 34 private banks are used. These data are taken from the Superintendence of Banks of Ecuador public information. As main conclusions, it can be mentioned that i) there is evidence that the banking market in Ecuador has an oligopolistic structure, ii) the banking sector would operate as if there were 7 companies in the entire market, and could be classified in large and medium banks, and iii) the results of the Herfindahl-Hirschman show a moderate concentration along the analyzed period of loans and deposits, which does not rule out the option that the 4 biggest banks applied some market power.
\end{abstract}

Keywords: Market concentration, competition, banks, market power, deposits, loans 


\section{INTRODUCCIÓN}

En los últimos 15 años, el Ecuador y el sector bancario ha experimentado cambios estructurales significativos que han generado un proceso firme de consolidación después de los graves problemas ocasionados en el año 1999 por la crisis bancaria y el proceso de dolarización de la economía dado la desregulación que se dio en esa década. Esta desregulación fue causada a partir de las políticas neoliberales impuestas desde las cartas de intención del Fondo Monetario Internacional (FMI) y dado que el Banco Central del Ecuador no fijaba tasas máximas de interés para los productos financieros que se ofertaban en el sector.

En el Ecuador existe una entidad gubernamental que regula las diferentes acciones de los bancos públicos y privados denominada Superintendencia de Bancos del Ecuador (SB) además de otra entidad que se encarga de la regulación de los mercados para garantizar una competencia leal entre las empresas que constituyen algún sector en la economía y previniendo el abuso de poder de mercado de los operadores económicos desde el año 2012, la Superintendencia de Control y Poder de Mercado (SCPM).

Este estudio utiliza indicadores de concentración de mercado para medir la competencia en el sector bancario privado ecuatoriano desde el año 2000 hasta el 2015 en dos principales indicadores bancarios: cartera y depósitos, dado que son una proxy de ventas en este sector, con el objetivo de: en primer lugar, analizar la evolución de la estructura de mercado, en segundo lugar determinar el nivel de competencia y de concentración del mercado.

La competencia en los diferentes sectores de una economía ayuda a aumentar el bienestar social ya que reduce precios y contrae los excedentes del productor, además genera mejoras en los productos o servicios proporcionados a los consumidores. En el sector bancario ocurre algo similar desde el punto de vista de la tasa de interés que sería el precio del producto, y ante una mayor competencia se esperaría una tasa de interés más baja. La importancia de la medición de la concentración de mercado encuentra una justificación teórica llamada paradigma estructura-conducta-resultado Bain (1951), la cual postula que pocas y grandes empresas (alta concentración) son más fáciles de comprometerse en conductas anticompetitivas (colusión).

La medición de los indicadores de concentración ayudará a determinar si el mercado bancario o sistema financiero nacional se encuentra en una situación de monopolio o de competencia perfecta en los diferentes años de estudio.

El sistema bancario privado en Ecuador desde la dolarización ha encontrado un proceso de consolidación del mismo, que lo ha vuelto más sólido, solvente y líquido, (Superintendencia de Bancos del Ecuador, 2016), sin embargo este proceso de consolidación puede haber generado un relajamiento en la competencia entre los bancos. En el Ecuador existe una premisa sobre un sector bancario con características oligopolísticas y con un alto poder de mercado ya que existen 4 bancos considerados grandes que se espera que tengan una alta participación de mercado .

En la actualidad muy pocas investigaciones en Ecuador han abordado 
este tema, que busca determinar cómo está estructurada la banca privada y bajo qué características compiten estos bancos, la más relevante es la de Salgado (2010) quien expone que la banca privada maneja aproximadamente las dos terceras partes de los activos y depósitos del sistema financiero regulado, seguida a mucha distancia por la banca pública que aporta alrededor del $13,2 \%$ y que los bancos privados son los intermediarios financieros que promueven la mayor concentración del crédito. Según conocimiento de los autores, ningún estudio ha sido publicado, por lo que el propósito de esta investigación es proporcionar información a la sociedad del ambiente competitivo en el que opera este sector en el Ecuador.

Por otro lado, se busca que este estudio sirva como herramienta de política pública a las instituciones que se encargan de regular el mercado, de tal manera que puedan ser aplicadas las propuestas a las que se llega por medio de las conclusiones de este estudio.

\section{ESTRUCTURA Y COMPETENCIA DEL SISTEMA BANCARIO ECUATO- RIANO.}

El sistema financiero ecuatoriano está conformado por entidades públicas y privadas debidamente autorizadas y reguladas por la SB que operan en la intermediación financiera, a través de los depósitos para que estos luego sean utilizados en operaciones de crédito y de inversión.

Según la regulación ecuatoriana por medio de la Superintendencia de Bancos del Ecuador (2015) el sistema financiero del Ecuador está compuesto por bancos sean estos públicos y/o privados, sociedades financieras, cooperativas de ahorro y crédito y mutualistas. Este sector es fundamental en las operaciones de la economía ya que generan financiamiento a sectores productivos del país, incrementando la riqueza.

Para este trabajo se ha realizado un análisis de los bancos privados ya que son los que proporcionan mayor información al día de sus operaciones ante la entidad de control, por otro lado se ha excluido las cooperativas de ahorro y crédito ya que son reguladas por otra entidad debido al comportamiento de su mercado relevante que tiene dos puntos de vista: i) producto y ii) dimensión geográfica; estas dos divisiones de mercado permiten identificar a los competidores reales de empresas afectadas que puedan limitar el comportamiento de estas o limitar su actuación dentro del marco de una competencia efectiva.

En el Ecuador, la SB realiza una clasificación anual de tamaño de bancos, en el cual determinan esta medición en base al tamaño de los activos de cada institución. Hasta diciembre del 2015 la SB clasificó a los bancos de la siguiente manera: Banco Pichincha, Banco Guayaquil, Banco del Pacifico y Produbanco como bancos grandes; Banco del Austro, Banco Bolivariano, Citibank, Banco General Rumiñahui, Banco Internacional, Banco de Loja, Banco Machala, Banco Solidario y Banco Procredit como bancos medianos; y Banco Amazonas, Banco Comercial de Manabí, Banco del Litoral, Banco CoopNacional, Banco Capital, Banco Finca, Banco DelBank, Banco D-Miro, 
Banco Desarrollo como bancos pequeños. Los bancos mencionados son el total de bancos que conforman el sistema bancario privado nacional del Ecuador .

La existencia de una competencia efectiva entre las empresas constituye uno de los elementos definitorios de la economía de mercado, disciplina la actuación de las empresas y reasigna los recursos productivos en favor de los operadores o las técnicas más eficientes. Esta eficiencia productiva se traslada al consumidor en forma de menores precios (tasas de interés) o de un aumento de la cantidad ofrecida de los productos, de su variedad y calidad, con el consiguiente incremento del bienestar del conjunto de la sociedad (Preámbulo, Ley 15/2007 de Defensa de la Competencia de España).

El presente estudio se limita a analizar el mercado relevante de productos, desde el punto de vista de la cartera y depósitos. El mercado de productos de referencia comprende la totalidad de los productos y servicios que los consumidores consideren intercambiables o sustituibles en razón de sus características, su precio o el uso que se prevea hacer con ellos.

En la Tabla 1 que se muestra a continuación se observa la participación en el total de cartera de la banca privada desde el año 2000 hasta el 2015, mostrando lo descrito anteriormente sobre la clasificación de tamaño de bancos, donde los 4 bancos privados más grandes poseen la mayor participación de mercado en la cuenta de cartera, seguido por 8 bancos de clasificación mediana para el año 2015. Por tanto, esta información nos muestra algo muy importante en la cuenta de cartera, existen cuatro bancos líderes y el resto de bancos podríamos mencionar como posibles seguidores.

Existe una competencia interna muy fuerte dentro de las tres clasificaciones de la SB, dado que año a año existe una diferencia en media muy grande entre grupos; para el año 2015 la participación media de cartera de los bancos privados grandes es de $16,4 \%$ mientras que apenas $3,5 \%$ y $0,3 \%$ de los bancos privados medianos y pequeños respectivamente. 
Tabla 1: Participación de la cartera (créditos) total del sistema bancario privado por institución

\begin{tabular}{|c|c|c|c|c|c|c|c|c|c|c|c|c|c|c|c|c|}
\hline $\begin{array}{ll}\text { BANCOS } & \text { AÑOS } \\
\end{array}$ & 2000 & 2001 & 2002 & 2003 & 2004 & 2005 & 2006 & 2007 & 2008 & 2009 & 2010 & 2011 & 2012 & 2013 & 2014 & 2015 \\
\hline PICHINCHA & 18,8 & 23,8 & 26,0 & 25,7 & 24,1 & 26,0 & 26,2 & 28,4 & 31,4 & 30,0 & 30,4 & 28,3 & 29,1 & 29,7 & 29,9 & 28,6 \\
\hline PACIFICO & 9,7 & 6,1 & 5,8 & 5,4 & 7,0 & 7,8 & 8,9 & 8,3 & 8,5 & 8,4 & 10,0 & 10,9 & 11,5 & 11,5 & 12,2 & 15,1 \\
\hline GUAYAQUIL & 10,6 & 12,2 & 13,5 & 12,5 & 11,7 & 10,8 & 11,1 & 11,2 & 11,7 & 12,8 & 12,8 & 12,7 & 12,3 & 11,9 & 12,1 & 11,1 \\
\hline PRODUBANCO & 8,2 & 11,5 & 12,2 & 11,3 & 10,6 & 9,8 & 8,9 & 8,7 & 7,1 & 8,4 & 7,9 & 8,1 & 8,2 & 9,0 & 11,2 & 10,8 \\
\hline INTERNACIONAL & 3,3 & 6,2 & 7,8 & 8,1 & 7,9 & 8,5 & 8,7 & 8,8 & 8,0 & 8,6 & 7,8 & 7,7 & 7,6 & 8,3 & 8,3 & 8,9 \\
\hline BOLIVARIANO & 5,5 & 7,7 & 9,8 & 9,6 & 8,5 & 7,8 & 7,6 & 7,6 & 7,5 & 7,4 & 7,4 & 7,5 & 7,2 & 7,3 & 7,7 & 7,7 \\
\hline AUSTRO & 2,6 & 2,8 & 3,0 & 3,5 & 4,1 & 4,0 & 4,0 & 4,5 & 4,6 & 4,3 & 5,0 & 4,9 & 4,8 & 4,8 & 4,8 & 4,8 \\
\hline SOLIDARIO & 1,9 & 2,6 & 3,6 & 4,1 & 4,3 & 3,9 & 3,6 & 3,0 & 2,5 & 2,1 & 1,7 & 1,7 & 1,7 & 3,1 & 2,9 & 2,8 \\
\hline MACHALA & 1,4 & 2,0 & 2,5 & 2,9 & 2,7 & 2,7 & 2,7 & 2,5 & 2,1 & 2,3 & 2,2 & 2,3 & 2,2 & 2,1 & 2,0 & 1,9 \\
\hline GENERAL RUMIÑAHUI & 1,4 & 2,7 & 3,5 & 3,3 & 3,0 & 2,8 & 2,7 & 2,2 & 2,2 & 2,1 & 1,9 & 2,1 & 2,1 & 2,0 & 2,0 & 1,9 \\
\hline PROCREDIT & 0 & 0 & 0 & 0 & 1,1 & 1,4 & 1,7 & 2,5 & 2,5 & 2,5 & 2,3 & 2,4 & 2,3 & 1,9 & 1,5 & 1,4 \\
\hline CITIBANK & 1,9 & 1,2 & 1,7 & 1,3 & 1,6 & 1,1 & 1,1 & 1,1 & 1,2 & 1,2 & 1,4 & 1,7 & 1,8 & 1,7 & 1,5 & 1,4 \\
\hline LOJA & 0,6 & 1,0 & 1,0 & 1,1 & 1,4 & 1,3 & 1,3 & 1,4 & 1,4 & 1,2 & 1,4 & 1,6 & 1,5 & 1,4 & 1,4 & 1,3 \\
\hline BANCODESARROLLO & 0 & 0 & 0 & 0 & 0 & 0 & 0 & 0 & 0 & 0 & 0 & 0 & 0 & 0 & 0,5 & 0,6 \\
\hline D-MIRO S,A, & 0 & 0 & 0 & 0 & 0 & 0 & 0 & 0 & 0 & 0 & 0 & 0,3 & 0,3 & 0,3 & 0,4 & 0,5 \\
\hline AMAZONAS & 0,8 & 1,1 & 1,0 & 1,1 & 1,3 & 1,1 & 1,1 & 0,8 & 0,7 & 0,7 & 0,5 & 0,4 & 0,4 & 0,5 & 0,5 & 0,4 \\
\hline CAPITAL & 0 & 0 & 0 & 0 & 0 & 0 & 0 & 0,6 & 0,5 & 0,3 & 0,4 & 0,5 & 0,5 & 0,5 & 0,5 & 0,3 \\
\hline FINCA & 0 & 0 & 0 & 0 & 0 & 0 & 0 & 0 & 0,3 & 0,3 & 0,2 & 0,3 & 0,3 & 0,2 & 0,2 & 0,2 \\
\hline COMERCIAL DE MANABI & 0,1 & 0,2 & 0,2 & 0,2 & 0,2 & 0,2 & 0,2 & 0,2 & 0,1 & 0,1 & 0,1 & 0,1 & 0,1 & 0,1 & 0,1 & 0,1 \\
\hline COOPNACIONAL & 0 & 0 & 0 & 0 & 0 & 0 & 0 & 0 & 0 & 0 & 0 & 0,2 & 0,2 & 0,1 & 0,1 & 0,1 \\
\hline DELBANK & 0 & 0 & 0 & 0 & 0 & 0,0 & 0,1 & 0,1 & 0,1 & 0,1 & 0,1 & 0,1 & 0,1 & 0,1 & 0,1 & 0,1 \\
\hline LITORAL & 0,1 & 0,1 & 0,2 & 0,2 & 0,2 & 0,2 & 0,2 & 0,1 & 0,1 & 0,1 & 0,1 & 0,1 & 0,1 & 0,1 & 0,1 & 0,1 \\
\hline FILANBANCO & 24,8 & 11,2 & 0 & 0 & 0 & 0 & 0 & 0 & 0 & 0 & 0 & 0 & 0 & 0 & 0 & 0 \\
\hline ABNAMRO & 2,8 & 0 & 0 & 0 & 0 & 0 & 0 & 0 & 0 & 0 & 0 & 0 & 0 & 0 & 0 & 0 \\
\hline ASERVAL & 1,5 & 0 & 0 & 0 & 0 & 0 & 0 & 0 & 0 & 0 & 0 & 0 & 0 & 0 & 0 & 0 \\
\hline CENTROMUNDO & 1,1 & 1,5 & 1,8 & 2,5 & 2,7 & 2,1 & 1,8 & 0 & 0 & 0 & 0 & 0 & 0 & 0 & 0 & 0 \\
\hline UNIBANCO & 0,9 & 2,4 & 2,4 & 2,5 & 2,7 & 3,2 & 2,9 & 2,5 & 2,0 & 1,9 & 2,2 & 2,0 & 1,8 & 0 & 0 & 0 \\
\hline COFIEC & 0,7 & 0,5 & 0,4 & 0,3 & 0,3 & 0,2 & 0,2 & 0,1 & 0,1 & 0,1 & 0,1 & 0,1 & 0,1 & 0,1 & 0,0 & 0 \\
\hline TERRITORIAL & 0,3 & 0,1 & 0,1 & 0,3 & 0,3 & 0,6 & 0,7 & 0,7 & 0,6 & 0,9 & 0,6 & 0,7 & 0,6 & 0 & 0 & 0 \\
\hline SUDAMERICANO & 0,0 & 0,1 & 0,1 & 0,1 & 0,1 & 0,0 & 0,0 & 0,0 & 0,0 & 0,0 & 0,0 & 0,0 & 0,0 & 0,0 & 0 & 0 \\
\hline LLOYDS BANK(BLSA) & 1,2 & 2,0 & 1,8 & 1,8 & 1,3 & 0,8 & 1,1 & 1,4 & 1,4 & 0 & 0 & 0 & 0 & 0 & 0 & 0 \\
\hline $\begin{array}{l}\text { M y M JARAMILLO } \\
\text { ARTEAGA }\end{array}$ & 0 & 0,9 & 1,7 & 2,2 & 2,8 & 3,2 & 3,3 & 3,4 & 3,4 & 0 & 0 & 0 & 0 & 0 & 0 & 0 \\
\hline ANDES & 0 & 0 & 0 & 0 & 0,3 & 0,3 & 0 & 0 & 0 & 0 & 0 & 0 & 0 & 0 & 0 & 0 \\
\hline PROMERICA & 0 & 0 & 0 & 0 & 0 & 0 & 0 & 0 & 0 & 2,8 & 3,2 & 3,1 & 3,1 & 3,1 & 0 & 0 \\
\hline
\end{tabular}

Fuente: Superintendencia de Bancos del Ecuador (2015) 
En la Tabla 2 se observa la participación en el total de depósitos de la banca privada desde el año 2000 hasta el 2015, mostrando así lo descrito anteriormente sobre la clasificación de tamaño de bancos y la participación de cartera en el mercado, donde los 4 bancos privados más grandes poseen la mayor participación de mercado en la cuenta de depósitos, seguido por 8 bancos de clasificación mediana para el año 2015.

Tabla 2: Participación de los depósitos totales del sistema bancario privado por institución

\begin{tabular}{|c|c|c|c|c|c|c|c|c|c|c|c|c|c|c|c|c|}
\hline BANCOS & 2000 & 2001 & 2002 & 2003 & 2004 & 2005 & 2006 & 2007 & 2008 & 2009 & 2010 & 2011 & 2012 & 2013 & 2014 & 2015 \\
\hline PICHINCHA & 23,7 & 29,5 & 28,1 & 27,8 & 11,5 & 24,2 & 13,1 & 27,1 & 27,6 & 28,1 & 28,2 & 28,2 & 28,9 & 28,9 & 29,9 & 29,8 \\
\hline PACIFICO & 12,9 & 2,6 & 7,8 & 8,6 & 12,6 & 10,9 & 5,9 & 10,8 & 10,5 & 10,8 & 11,4 & 12,3 & 12,2 & 12,8 & 11,9 & 12,7 \\
\hline PRODUBANCO & 10,1 & 11,9 & 11,3 & 11,1 & 10,1 & 10,1 & 4,9 & 9,2 & 10,2 & 10,1 & 9,7 & 9,0 & 9,6 & 9,8 & 12,4 & 12,4 \\
\hline GUAYAQUIL & 4,7 & 9,0 & 12,7 & 12,9 & 25,0 & 13,1 & 6,9 & 13,0 & 12,7 & 12,8 & 12,5 & 12,9 & 11,5 & 11,0 & 11,2 & 10,9 \\
\hline BOLIVARIANO & 4,3 & 7,3 & 8,6 & 8,2 & 8,0 & 8,4 & 2,1 & 8,4 & 8,5 & 8,1 & 8,5 & 8,3 & 8,5 & 8,7 & 8,8 & 9,0 \\
\hline INTERNACIONAL & 5,2 & 7,2 & 7,8 & 7,5 & 2,7 & 8,6 & 1,1 & 8,3 & 8,2 & 8,0 & 8,4 & 7,8 & 8,2 & 8,8 & 8,5 & 8,7 \\
\hline AUSTRO & 2,7 & 3,6 & 3,3 & 3,6 & 8,4 & 3,7 & 30,8 & 4,5 & 4,3 & 4,3 & 4,7 & 4,6 & 4,5 & 4,7 & 4,9 & 5,0 \\
\hline MACHALA & 1,2 & 2,0 & 2,4 & 2,7 & 2,6 & 2,7 & 4,1 & 2,5 & 2,4 & 2,4 & 2,4 & 2,4 & 2,4 & 2,4 & 2,3 & 2,0 \\
\hline GENERALRUMIÑAHUI & 1,9 & 3,6 & 2,7 & 2,6 & 2,8 & 2,2 & 1,2 & 2,2 & 2,3 & 2,3 & 2,1 & 2,1 & 2,0 & 2,0 & 2,0 & 1,9 \\
\hline SOLIDARIO & 0,4 & 0,7 & 2,4 & 2,5 & 2,2 & 2,5 & 1,4 & 1,6 & 1,2 & 1,2 & 1,0 & 1,0 & 1,0 & 1,9 & 1,7 & 1,7 \\
\hline CITIBANK & 4,2 & 4,9 & 3,7 & 2,9 & 3,7 & 2,3 & 4,2 & 1,9 & 2,1 & 1,7 & 2,1 & 1,8 & 1,9 & 1,8 & 1,8 & 1,5 \\
\hline LOJA & 0,8 & 1,3 & 1,0 & 1,1 & 0,4 & 1,2 & 0,0 & 1,4 & 1,4 & 1,5 & 1,5 & 1,5 & 1,4 & 1,4 & 1,4 & 1,4 \\
\hline PROCREDIT & 0 & 0 & 0 & 0 & 0,0 & 0,3 & 0,0 & 0,7 & 1,0 & 1,3 & 1,1 & 1,1 & 1,1 & 1,1 & 0,9 & 0,8 \\
\hline COOPNACIONAL & 0 & 0 & 0 & 0 & 0 & 0 & 0 & 0 & 0 & 0 & 0 & 0,6 & 0,6 & 0,6 & 0,5 & 0,6 \\
\hline AMAZONAS & 0,9 & 0,8 & 1,0 & 1,0 & 1,7 & 1,0 & 1,3 & 1,0 & 0,6 & 0,6 & 0,6 & 0,6 & 0,6 & 0,4 & 0,4 & 0,5 \\
\hline BANCODESARROLLO & 0 & 0 & 0 & 0 & 0 & 0 & 0 & 0 & 0 & 0 & 0 & 0 & 0 & 0 & 0,3 & 0,4 \\
\hline CAPITAL & 0 & 0 & 0 & 0 & 0 & 0 & 0 & 0,4 & 0,3 & 0,3 & 0,4 & 0,4 & 0,5 & 0,4 & 0,4 & 0,3 \\
\hline COMERCIAL DE MANABI & 0,2 & 0,3 & 0,1 & 0,1 & 0,9 & 0,2 & 0,6 & 0,2 & 0,3 & 0,2 & 0,2 & 0,2 & 0,2 & 0,2 & 0,1 & 0,1 \\
\hline LITORAL & 0,3 & 0,5 & 0,3 & 0,6 & 0,5 & 0,4 & 0,1 & 0,1 & 0,1 & 0,1 & 0,1 & 0,1 & 0,1 & 0,1 & 0,1 & 0,1 \\
\hline D-MIROS,A, & 0 & 0 & 0 & 0 & 0 & 0 & 0 & 0 & 0 & 0 & 0 & 0 & 0,0 & 0,1 & 0,1 & 0,1 \\
\hline DELBANK & 0 & 0 & 0 & 0 & 0 & 0,0 & 0,0 & 0,0 & 0,0 & 0,0 & 0,0 & 0,1 & 0,0 & 0,0 & 0,0 & 0,1 \\
\hline FINCA & 0 & 0 & 0 & 0 & 0 & 0 & 0 & 0 & 0,0 & 0,0 & 0,0 & 0,0 & 0,0 & 0,0 & 0,0 & 0,03 \\
\hline FILANBANCO & 18,7 & 11,1 & 0 & 0 & 0 & 0 & 0 & 0 & 0 & 0 & 0 & 0 & 0 & 0 & 0 & 0 \\
\hline ABNAMRO & 4,7 & 0 & 0 & 0 & 0 & 0 & 0 & 0 & 0 & 0 & 0 & 0 & 0 & 0 & 0 & 0 \\
\hline ASERVAL & 0,2 & 0 & 0 & 0 & 0 & 0 & 0 & 0 & 0 & 0 & 0 & 0 & 0 & 0 & 0 & 0 \\
\hline CENTROMUNDO & 0,0 & 0,1 & 1,3 & 1,6 & 1,3 & 1,6 & 0,6 & 0 & 0 & 0 & 0 & 0 & 0 & 0 & 0 & 0 \\
\hline UNIBANCO & 0,4 & 0,5 & 2,1 & 1,8 & 0,1 & 2,6 & 1,2 & 2,1 & 1,5 & 1,4 & 1,4 & 1,4 & 1,3 & 0 & 0 & 0 \\
\hline COFIEC & 0,1 & 0,1 & 0,1 & 0,0 & 1,0 & 0,0 & 0 & 0,0 & 0,0 & 0,0 & 0,1 & 0,2 & 0,1 & 0,1 & 0,1 & 0 \\
\hline TERRITORIAL & 0,1 & 0,1 & 0,1 & 0,1 & 0,1 & 0,4 & 0,5 & 0,5 & 0,6 & 0,8 & 0,5 & 0,6 & 0,5 & 0 & 0 & 0 \\
\hline SUDAMERICANO & 0,0 & 0,0 & 0,0 & 0,0 & 0,1 & 0,0 & 0,6 & 0,0 & 0,0 & 0,0 & 0,0 & 0,0 & 0,0 & 0,0 & 0 & 0 \\
\hline LLOYDSBANK(BLSA) & 2,4 & 2,8 & 1,7 & 1,3 & 0,2 & 0,8 & 0,1 & 1,1 & 1,1 & 0 & 0 & 0 & 0 & 0 & 0 & 0 \\
\hline M,M,JARAMILLOARTEAGA & 0 & 0,3 & 1,4 & 1,8 & 2,4 & 2,6 & 1,3 & 3,1 & 3,0 & 0 & 0 & 0 & 0 & 0 & 0 & 0 \\
\hline ANDES & 0 & 0 & 0 & 0 & 1,6 & 0,3 & 17,9 & 0 & 0 & 0 & 0 & 0 & 0 & 0 & 0 & 0 \\
\hline PROMERICA & 0 & 0 & 0 & 0 & 0 & 0 & 0 & 0 & 0 & 3,1 & 3,1 & 2,8 & 2,7 & 2,8 & 0 & 0 \\
\hline
\end{tabular}


La información descrita en la Tabla 2 indica la competencia de la banca privada en la cuenta de depósitos y un posible comportamiento de los 4 bancos más grandes como "líderes" y el resto como "seguidoras" dada su cuota de mercado. También se observa que existe una competencia muy fuerte dentro de las tres clasificaciones de la SB al igual que en la cuenta cartera, dado que año a año existe una diferencia en media muy grande entre grupos; para el año 2015 la participación media de los depósitos de los bancos privados grandes es de $16,4 \%$ mientras que apenas 3,6\% y $0,2 \%$ de los bancos privados medianos y pequeños respectivamente.

\section{MATERIALES Y MÉTODOS}

La concentración en un mercado es la medida de estructura de competencia más importante, por lo tanto es muy necesario saber el número de empresas que compiten en el mercado, y además su tamaño relativo ya que esta ayuda a mejorar el uso de herramientas para la política de competencia y regulación de los mercados (Compte, Jenny, \& Rey, 2002; Tirole, 1998; Bain, 1954).

Se espera que exista mayor poder de mercado si: i) solo hay una o pocas empresas en el mercado, ii) muchas empresas, pero existe un número reducido de ellas que son muy grandes en relación al resto (empresa dominante). Weisman (2005) señala que a medida que aumenta la concentración de mercado, la competencia y la eficiencia disminuyen y la probabilidad de colusión y monopolio aumentan.

En algunos estudios referente a la concentración del sector bancario se demuestra que este sector se encuentra entre media-alta concentración: Caso OCDE (Cetorelli, 2001), Caso EEUU donde una mayor concentración del sector bancario implica una menor entrada de empresas y una mayor salida de empresas antiguas (Cetorelli, 2003), Caso Italia (Cetorelli, 1999), Caso economías emergentes donde se demuestra evidencia positiva entre competencia y estabilidad del sistema bancario (Beck, 2008), Caso España (Pueyo Sánchez, 2003).

A este respecto Gonzales Martínez (2008) al revisar casos de economías similares a la de Ecuador, muestra el Caso Bolivia donde el mercado bancario de este país tiene una estructura oligopolística en el que destacan unas pocas instituciones grandes entre varias medianas y pequeñas, además se muestra una elevada concentración en activos y depósitos; por otro lado, para el caso guatemalteco existe evidencia de encontrarse en una competencia perfecta en el mercado de créditos tanto en bancos grandes como pequeños, sin embargo cuando este se analiza por medio del índice Herfindahl-Hirschman (HHI) muestra una estructura de competencia oligopolística (Díaz Castellanos, 2010). En Perú un estudio sobre poder de mercado de la banca, muestra que efectivamente existe un ligero poder de mercado de parte de ciertos bancos grandes y que esto afecta a los márgenes de intermediación financiera (Sánchez, 2013). Sin embargo en Colombia un estudios muestra que se 
presentaron mejorías en términos de estabilidad financiera a medida que el sistema bancario se concentró durante las 2 décadas más recientes; sin embargo, dicha relación no es lineal, y es posible encontrar un nivel óptimo de concentración (Mosquera \& Gómez, 2013). En Venezuela se analizó el comportamiento del índice de concentración bancaria para colocaciones (créditos), depósitos y patrimonio entre 1993 - 2004 mediante el HHI y se concluyó que existe un mercado moderadamente concentrado (Borgucci, 2008).

Existen algunas medidas para calcular la concentración en un mercado, pero las más usadas y recomendadas por (Organization for Economic Cooperation and Development (OECD), 1993) son: Ratio de Concentración (CRn) y el Índice de Herfindahl-Hirschman (HHI).Estas medidas describen la relación entre el porcentaje acumulado de producción y el número acumulado de empresas en la industria, ordenadas de acuerdo a su producción o venta.

Para este trabajo se utilizan el CR4 y el HHI ya que son los indicadores de concentración y competencia de mercado más utilizados en el enfoque Estructura-Comportamiento-Resultado (ECD) de forma estructural o informal; este enfoque mediante los índices de estudio propuestos, determina que mientras más alto es el índice más alta es la concentración, Además el HHI ayuda a mostrar el grado de concentración o de competencia en un sector de manera más global ya que toma en cuenta las participaciones de mercado y el número de empresas (Uzcategui \& Camino, 2017).

Ratio de Concentración (CRn), Puede ser calculado a un nivel de (m) empresas, donde $\mathrm{m}$ es el número que se define para las mayores empresas que se desean analizar (ordenadas por cuota de mercado (S_i)),

$$
C R_{m}=\sum_{i=1}^{m} s_{i}
$$

Mientras más cerca de 1 es el ratio de concentración las (m) empresas acumulan la mayor cuota de mercado de la industria, Una de las ventajas que posee este ratio es que solo se necesita la información de las (m) empresas más grandes (Hannaford, 2007), Si el índice de concentración es 1 se asume que la industria se comporta como un monopolio puro o una posición monopolística en términos de cuota de mercado de las empresas de la observación, Cuando el valor del índice es mayor o igual a 0,75 el sector se encuentra en una posición oligopolística o altamente concentrado,

Índice de Herfindahl-Hirschman (HHI). Es la suma de los cuadrados de las cuotas de mercado de todas las empresas de una industria (U.S Department of Justice and the Federal Trade Commission, 2010).

$$
H H I=\sum_{i=1}^{m} S_{i}^{2}
$$


Las cuotas de mercado se miden en términos porcentuales y el valor máximo que puede tomar el índice HHI es de 10,000, una de las ventajas es que tiene en cuenta a todas las empresas de la industria. La U.S Department of Justice and the Federal Trade Commission (2010) ha definido las siguientes escalas del índice HHI:

- HHI inferior a 0,01, el mercado es altamente competitivo,

- HHI inferior a 0,15, el mercado no está concentrado,

- HHI entre 0,15 y 0,25, el mercado está moderadamente concentrado,

- HHI mayor a 0,25, el mercado está altamente concentrado,

Otra clasificación propuesta por la European Commission (2004) indica que el HHI puede ser explicado en una escala diferente:

- HHI inferior a 1000, el mercado no está concentrado

- HHI entre 1000 y 2000, el mercado está moderadamente concentrado,

- HHI mayor a 2000, el mercado está altamente concentrado,

Número Equivalente (n*). Una vez calculado el Índice de Herfindahl-Hirschman, este indicador sirve para poder calcular lo que se denomina Número Equivalente que muestra el número de empresas de igual tamaño que daría lugar a una industria con un HHI determinado.

$$
n^{*}=\frac{1}{H H I}
$$

\section{RESULTADOS Y DISCUSIÓN}

Tabla 3: Índices de concentración del sistema bancario

\begin{tabular}{|l|cc|cc|cc|c|}
\hline AÑO & HHI CARTERA & HHI DEPOSITOS & n* CARTERA & n* DEPOSITOS & Cr4 CARTERA & Cr4 DEPOSITOS & \#BANCOS \\
\hline 2000 & 1314,59 & 1304,01 & 8 & 8 & 63,87 & 65,31 & 24 \\
2001 & 1151,48 & 1392,23 & 9 & 7 & 58,70 & 61,49 & 23 \\
2002 & 1258,71 & 1329,18 & 8 & 8 & 61,45 & 60,65 & 22 \\
2003 & 1205,33 & 1316,07 & 8 & 8 & 59,12 & 60,39 & 22 \\
2004 & 1094,30 & 1206,91 & 9 & 8 & 54,86 & 59,14 & 24 \\
2005 & 1162,90 & 1177,86 & 9 & 8 & 55,15 & 58,28 & 25 \\
2006 & 1177,36 & 1596,97 & 8 & 6 & 55,14 & 68,68 & 24 \\
2007 & 1281,48 & 1297,75 & 8 & 8 & 57,03 & 60,05 & 24 \\
2008 & 1433,70 & 1327,89 & 7 & 8 & 59,64 & 60,98 & 25 \\
2009 & 1389,30 & 1352,69 & 7 & 7 & 59,79 & 61,78 & 25 \\
2010 & 1429,43 & 1370,94 & 7 & 7 & 61,14 & 61,78 & 24 \\
2011 & 1327,10 & 1375,23 & 8 & 7 & 60,01 & 62,45 & 26 \\
2012 & 1366,03 & 1396,75 & 7 & 7 & 61,03 & 62,19 & 26 \\
2013 & 1420,21 & 1422,65 & 7 & 7 & 62,10 & 62,60 & 24 \\
2014 & 1489,41 & 1511,36 & 7 & 7 & 62,10 & 65,46 & 23 \\
2015 & 1468,03 & 1519,33 & 7 & 7 & 65,39 & 65,77 & 22 \\
\hline
\end{tabular}

Fuente: Superintendencia de Bancos del Ecuador (2015) 


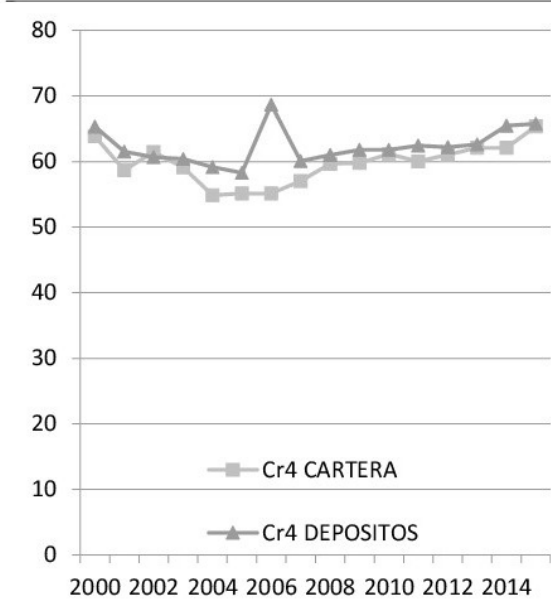

Figura 1: Índice de Concentración 4

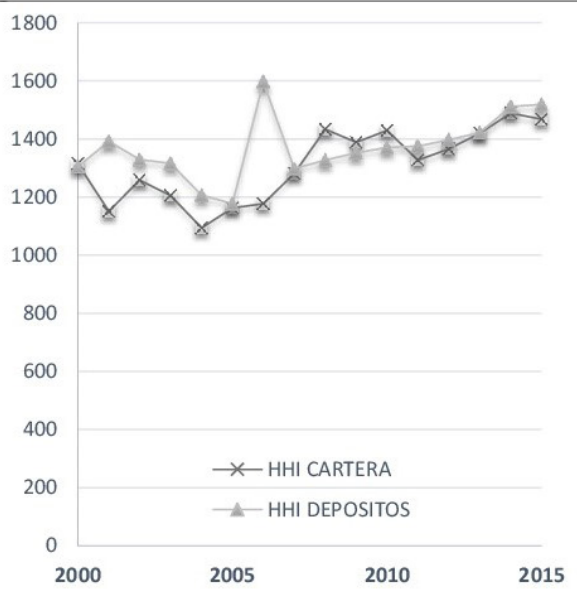

Figura 2: Índice Herfindahl-Hirschman

En la Tabla 3 se puede observar los diferentes resultados de concentración del sector bancario en Ecuador, además del número de bancos en cada año de análisis; los índices de concentración analizados son el de Herfindahl-Hirschman (HHI), número equivalente (n*) y ratio de concentración 4 (Cr4) de los depósitos bancarios y cartera o créditos concedidos por el sector

La información obtenida de la tabla 3 y de las figuras 1 y 2 muestran que el sector bancario en Ecuador desde el año 2001 hasta el año 2005 presentó un decrecimiento en sus índices de concentración tanto en depósitos como en cartera, y desde el año 2006 en adelante estos mismos índices de concentración comenzaron a crecer hasta el año 2015, observando que los dos últimos años no han variado de forma significativa. El número de bancos en todo el sector bancario del 2000 al 2015 ha decrecido en un 8,33\% lo que se podría interpretar como un sector donde existen barreras de entrada a nuevos bancos dado un posible comportamiento oligopolístico.

El índice de Herfindahl-Hirschman (HHI) a diciembre del año 2015 es igual a 1468 (cartera) y 1519 (depósitos), mostrando un comportamiento de mercado moderadamente concentrado tanto en cartera como en depósitos, en este análisis se excluyen a la banca pública y cooperativas de ahorro y crédito (entidades microfinancieras). Históricamente desde el año 2000 hasta diciembre del 2015 este índice se encontró entre 1094,3 y 1596,97 puntos, existiendo una concentración moderada en todos los periodos. Estos resultados van en concordancia con estudios para economías similares de la región tales como el caso de Venezuela, Perú, Bolivia, Colombia y Guatemala, donde se concluye que el mercado bancario se encuentra concentrado de manera moderada en un análisis de tiempo de similar magnitud. 
El ratio de concentración de las 4 primeras empresas ( $\mathrm{Cr} 4)$ en base a su participación de mercado en cartera y depósitos es de 65,39\% y 65,77\% respectivamente para diciembre del 2015, estos valores demuestran un comportamiento oligopolístico en las que las 4 empresas más grandes controlan el $65 \%$ del mercado de los depósitos y cartera.

El número equivalente $\left(n^{*}\right)$ muestra que si el sector bancario estuviera equitativamente distribuido, estaría conformado por 7 bancos desde el año 2012 hasta el 2015 tanto en cartera como en depósitos. En promedio, si las participaciones del mercado bancario hubieran estado igualmente distribuidas, este mercado habría estado conformado por 7 bancos.

De los resultados se obtiene evidencia que cuando existe un mayor índice de concentración de mercado, el número equivalente de empresas en dicho mercado tiende a disminuir, es decir dicho sector se empieza a comportar de forma oligopolística que si no se regula a tiempo y con las medidas adecuadas podría formarse un mercado altamente concentrado que perjudique al consumidor.

\section{DISCUSIÓN Y CONCLUSIONES}

SEl sector bancario en el Ecuador desde el año 2000 hasta el 2015 muestra valores altos de los diferentes ratios de concentración tanto para cartera y depósitos, por tanto, existe evidencia de que dicho mercado en Ecuador posee una estructura oligopolística en donde existen 4 empresas (bancos) que controlan el 65\% del mercado desde el año 2012 hasta la actualidad, además el sector bancario operaría como si existiesen 7 empresas en todo el mercado, y se las podría clasificar en bancos grandes (4) y medianos (3) de los más fuertes. Los resultados del índice de Herfindahl-Hirschman muestran una concentración moderada a lo largo del periodo analizado en cartera y en depósitos, lo que no descarta la opción que los 4 bancos más grandes puedan ejercer cierto poder de mercado para elevar los precios de los productos financieros por encima de niveles competitivos.

De hecho, que este sector no se encuentre altamente concentrado sino que, de una forma moderada, responde a una mejora en la regulación del sistema financiero y que beneficia especialmente a los consumidores de los productos financieros de la banca, de tal manera que las tasas de interés, servicios financieros, servicios no operacionales no se encuentren con un costo muy elevado de parte de ciertas instituciones y que no perjudiquen el bienestar social de los individuos desde la perspectiva de un apoderamiento del excedente del consumidor.

Los resultados obtenidos van en concordancia con estudios de concentración del sector bancario para economías similares de la región, además muestra que existen bancos denominados grandes que pueden ejercer cierto poder de mercado.

Como no se descarta la opción de ciertos comportamientos de poder de mercado e inclusive colusivos, es importante mencionar que una mayor regulación prudencial de parte de la SB evitaría una estructura de mercado 
oligopolística colusiva perfecta en donde las empresas dominantes actúen de manera conjunta para restringir la competencia,

\section{REFERENCIAS BIBLIOGRÁFICAS}

Bain, J. (1951). Relation of the profit rate to industry concentration: American manufacturing, 1936-1940. Quarterly Journal of Economics, 65(3), 293324.

Bain, J. (1954). Economies of scale, concentration, and the condition of entry in twenty manufacturing industries. The American Economic Review, 1539.

Beck, T. (2008). Bank competition and financial stability: friends or foes? World Bank Policy Research Working Paper Series 4656, The World Bank.

Borgucci, E. (2008). La concentración y la intermediación bancaria en Venezuela entre 1993 y 2004. Revista de Ciencias Sociales, 14(2).

Cetorelli, N. (1999). Competitive analysis in banking: Appraisal of the methodologies. Economic Perspectives-Federal Reserve Bank of Chicago, 2-15.

Cetorelli, N. (2001). Does Bank Concentration Lead to Concentration in Industrial Sectors? Federal Reserve Bank of Chicago, 23, 2-15.

Cetorelli, N. (2003). Life-Cycle Dynamics in Industrial Sectors. The Role of Banking Market Structure. Quarterly Review, Federal Reserve Bank of St. Louis, 135-47.

Compte, O., Jenny, F., \& Rey, P. (2002). Capacity constrainsts, mergers and collusion. European Economic Review, 46(1), 1-29.

Díaz Castellanos, G. (2010). Estructura y poder del sector bancario de Guatemala. Gerencialidad Socio-empresarial.

European Commission. (2004). Guidelines on the Assessment of Horizontal Mergers. Brussels, Belgium: Official Journal of European Union.

Gonzales Martínez, R. (2008). Estructura de mercado, condiciones de entrada y número óptimo de bancos en el sistema bancario boliviano: Una aproximación de indicadores de concentración y movilidad intra-industrial. MPRA Paper No. 14012.

Hannaford, S. (2007). Market domination!: The impact of industry consolidation on competition, innovation, and consumer choice. Westport, United States: Greenwood Publishing Group.

Mosquera, M., \& Gómez, N. (2013). ¿Qué tipo de relación existe en Colombia entre concentración bancaria y estabilidad financiera? Ensayos sobre Política Económica, 31(71), 36-53.

Organization for Economic Cooperation and Development, OECD. (1993). Glossary of Industrial Organization Economics and Competition Law. Organization for Economic.

Pueyo Sánchez, J. (2003). Oligopolio y competencia en la banca española del siglo XX: concentración económica y movilidad intra-industrial, 19221995. Revista de Historia Economica-Journal of Iberian and Latin American Economic History, 21(1), 147-195.

Salgado, W. (2010). Banca de desarrollo en el Ecuador. (N. Unidas, Ed.) San- 
tiago de Chile, Chile: CEPAL - Serie Financiamiento del desarrollo No. 232.

Sánchez, G. (2013). Poder de mercado, intermediación financiera y banca: un enfoque de organización industrial. Economía, 36(71), 75.

Uzcategui, C., \& Camino, S. (2017). Estructura de la competencia del sector tabacalero en España: cigarrillos y tabaco de liar. Ciencia Unemi, 10(22), 20-28.

Superintendencia de bancos (SB). (2015, diciembre 23). Superintendencia de bancos. Retrieved from Superintendencia de bancos: http://www.sbs. gob.ec/practg/sbs_index?vp_art_id=1\&vp_tip $=11 \& v p \_l a n g=1 \& v p \_b u s-$ $\mathrm{cr}=11 \# 133$

Superintendencia de Bancos del Ecuador. (2016). ANALISIS FINANCIERO: SISTEMA DE BANCOS PRIVADOS. Superintendencia de Bancos del Ecuador, Dirección Nacional de Estudios e Información. Quito: Superintendencia de Bancos del Ecuador.

Tirole, J. (1998). Theory of Industrial Organization. USA: Hamilton Printing.

U.S Department of Justice and the Federal Trade Commission. (2010). Horizontal Merger Guidelines. Washington, US: United States: Department of Justice and the Federal Trade Commission.

Weisman, D. (2005). Assessing Market Power: The Trade-off Between Market Concentration and Multi-Market Participation. Journal of Competition Law and Economics, 1(2), 339-354. 